\title{
A novel no-sensors 3D model reconstruction from monocular video frames for a dynamic environment
}

\author{
Ghada Mohamed Fathy ${ }^{\text {Corresp., }, 2, \text {, Hanan Ali Hassan }}{ }^{1}$, Walaa Sheta $^{1}$, Fatma Omara ${ }^{2,3}$, Emad Nabil ${ }^{2,4}$ \\ 1 Informatics Research Institute, City for Scientific Research and Technological Applications, SRTA-City, Alexandria, Egypt \\ 2 Department of Computer Science, Faculty of Computers and Artificial Intelligence, Cairo University, Giza, Egypt \\ 3 Faculty of Engineering, Heliopolis University, Cairo, Egypt \\ ${ }^{4}$ Computer Science Department, Faculty of Computer and Information Systems, Islamic University of Madinah, Madinah, Saudi Arabia \\ Corresponding Author: Ghada Mohamed Fathy \\ Email address: gfathy@srtacity.sci.eg
}

Occlusion awareness is one of the most challenging problems in several fields such as multimedia, remote sensing, computer vision, and computer graphics. Realistic interaction applications are suffering from dealing with occlusion and collision problems in a dynamic environment. Creating dense 3D reconstruction methods is the best solution to solve this issue. However,these methods have poor performance in practical applications due to the absence of accurate depth, camera pose, and object motion. This paper proposes a new framework that builds a full 3D model reconstruction that overcomes the occlusion problem in a complex dynamic scene without using sensors' data. Popular devices such as a monocular camera are used to generate a suitable model for video streaming applications. The main objective is to create a smooth and accurate 3D point-cloud for a dynamic environment using cumulative information of a sequence of RGB video frames. The framework is composed of two main phases. First, uses unsupervised learning technique to predict; scene depth, camera pose, and objects' motion from RGB monocular videos. Second, generates a frame-wise point cloud fusion to reconstruct a 3D model based on a video frame sequence. Several evaluation metrics are measured; Localization error, RMSE, and Fitness between ground truth (KITTI's sparse LiDAR points) and predicted point-cloud. Moreover, compared the framework with different widely used state-of-the-art evaluation methods such as MRE and Chamfer Distance. Experimental results showed that the proposed framework surpassed the other methods and proved to be a powerful candidate in 3D model reconstruction. 
1 A Novel no Sensors 3D Model Reconstruction from Monocular Video

2 Frames for a Dynamic Environment

3

4

5

6

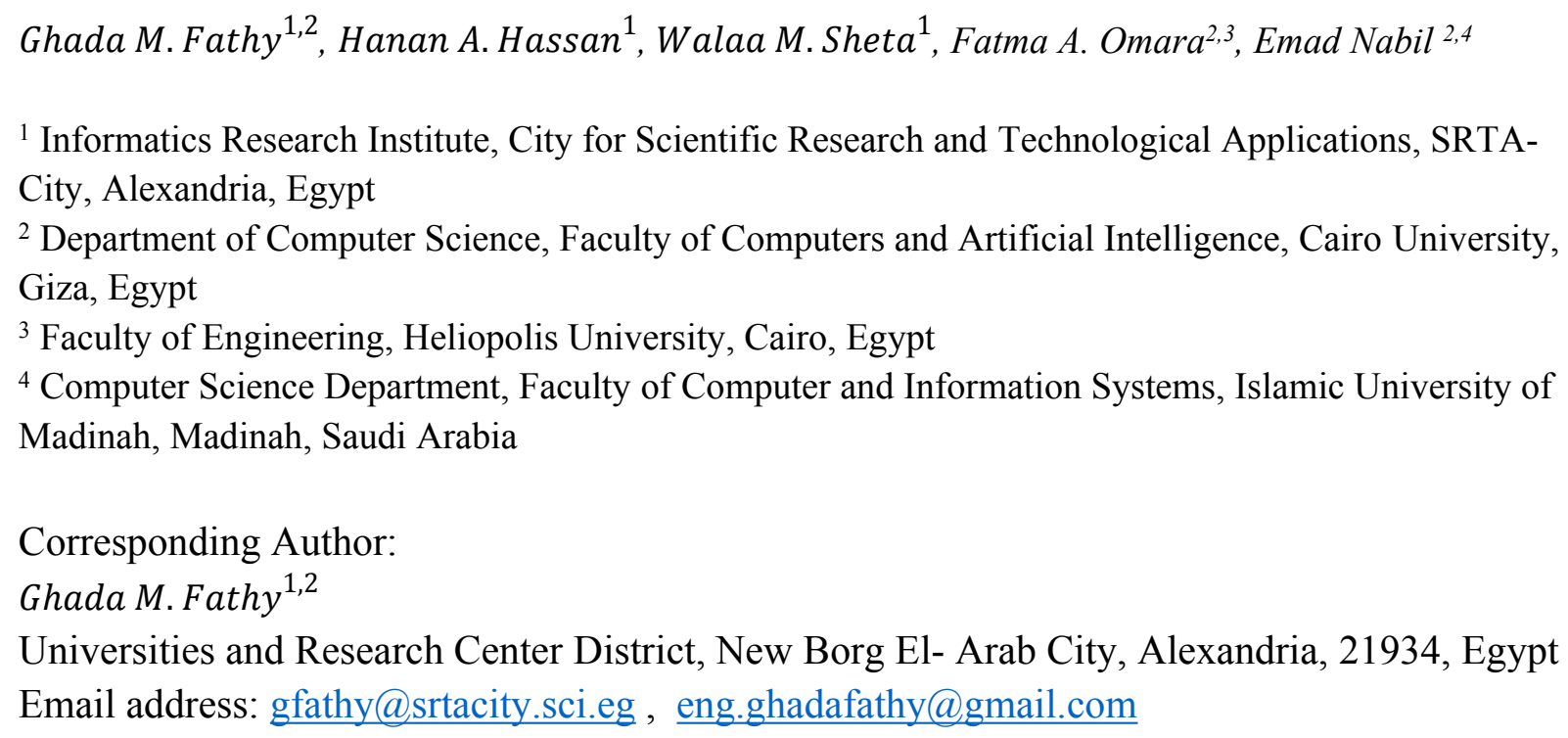

\section{Abstract}

Occlusion awareness is one of the most challenging problems in several fields such as multimedia, remote sensing, computer vision, and computer graphics. Realistic interaction applications are suffering from dealing with occlusion and collision problems in a dynamic environment. Creating dense 3D reconstruction methods is the best solution to solve this issue. However, these methods have poor performance in practical applications due to the absence of accurate depth, camera pose, and object motion. This paper proposes a new framework that builds a full 3D model reconstruction that overcomes the occlusion problem in a complex dynamic scene without using sensors' data. Popular devices such as a monocular camera are used to generate a suitable model for video streaming applications. The main objective is to create a smooth and accurate 3D pointcloud for a dynamic environment using cumulative information of a sequence of RGB video frames. The framework is composed of two main phases. First, uses unsupervised learning technique to predict; scene depth, camera pose, and objects' motion from RGB monocular videos. Second, generates a frame-wise point cloud fusion to reconstruct a 3D model based on a video frame sequence. Several evaluation metrics are measured; Localization error, RMSE, and Fitness between ground truth (KITTI's sparse LiDAR points) and predicted point-cloud. Moreover, compared the framework with different widely used state-of-the-art evaluation methods such as MRE and Chamfer Distance. Experimental results showed that the proposed framework surpassed the other methods and proved to be a powerful candidate in 3D model reconstruction.

\section{Introduction}


40 Constructing a full 3D model from a complex dynamic scene data has many applications in motion 41 capture, robot navigation, augmented reality, and autonomous driving. Moreover, it aims to 42 provide solutions to solve realistic interaction problems such as occlusion and collision. There are 43 many challenges to reconstruct 3D models from dynamic scenes, such as predict accurate depth 44 from sensors or a sequence of 2D RGB. To achieve that it needs to consider the camera pose, and 45 the motion of dynamic objects during navigation.

46 There are many techniques in computer vision that introduce different image-based 3D modeling techniques such as simultaneous location and mapping (SLAM) (Mur-Artal R. J., 2015), Multiview stereo (MVS) (Kuhn, 2019), photo tourism (Furukawa, 2009), and an RGB-D videobased method (Keller, 2013.). These methods use the point cloud representation to represent a realworld scene. A point cloud representation can be used for 3D inspection as it renders detailed 3D environments accurately. The depth camera such as (RGB-D) cameras, e.g., Microsoft Kinect, is widely used to reconstruct 3D indoor scenes (Chen, 2015). However, Kinect-like scanning devices fail to capture reliable depth images from outdoor scenes. Moreover, RGB-D cameras may not be readily available in most robotics and mobile devices, and it may also introduce sensors noise. A variant of solutions has been developed using different kinds of cameras, for example, monocular (Tateno, 2017) (Wang J. H., 2018), and Stereo (Hassan, 2017). Monocular cameras are most widely used because they are cheap, least restriction, and most ubiquitous for robots and mobile devices. However, the existing monocular 3D model reconstruction methods have poor performance due to the lack of accurate scene depth and camera pose.

Therefore, most reconstruction directions resort to predict depth and camera pose using learning techniques. Generating a 3D point cloud from learnt depth and learnt camera pose might be the right choice that solves the problem of using expensive sensors and gives accurate results in a dynamic scene. Recently, deep neural network has been used in learning and succeeded to predict depth from a single image (Liu F. C., 2015), (Laina, 2016), and (Casser, 2019.). One advantage of deep learning approaches is that the full scale can be predicted from a single image without the need of scene-based assumptions or geometric constraints. Nowadays, several realistic interaction applications still have limitations to deal with the occlusion problem in a real dynamic environment without using expensive sensors. one of the most effective solutions is to generate dense 3D reconstruction for the whole scene. However, the interaction in a dynamic environment requires a true depth map and explicit not only to detect the camera localization but also consider the moving objects into the scene with each other and with static objects in the background.

The main objectives of our framework are:

Creating a smooth and accurate 3D point-cloud for a dynamic environment using accumulative information from a sequence of RGB video frames. This method is used in solving several problems such as occlusion and collision. Due to the expensive cost of multi-sensors data, a monocular camera is used instead and compensated by unsupervised learning techniques to be suitable for video streaming applications. The framework consists of two stages. In the first stage, the online refinement process adapts new environment by integrating 3D motion of moving objects with depth and camera ego-motion. In the second stage, a full 3D model is reconstructed using frame-wise point cloud fusion. Figure 1 illustrates the proposed framework details. The rest of the 
82 paper is organized as follows: State of the art section describes the most relevant related work. The

83 proposed framework section presents an overview of the proposed approach. Monocular 3D Model 84 Reconstruction section, explains the Mathematical model for 3D model reconstruction. The 85 proposed 3D model reconstruction implementation section illustrated dataset, experiment metrics. 86 The Experimental results section prove the accuracy of the framework and give a comparison 87 between our method and the state-of-the-art techniques. Finally, conclusion and future work are 88 explained in the last section.

89 State of the Art

90 The development of the proposed framework has involved a review of research in the various 91 computer vision field. This section is oriented towards three main subjects: (1) Estimate depth 92 map from a single view. (2) Detect the camera position. (3) 3D reconstruction for a single object 93 or multiple objects (full 3D scene) in a static and dynamic environment.

Depth Estimation form single view, Scene depth estimation has gained increasing attention in the field of computer vision and robotics. Recently developed deep convolutional architectures for image-to-depth prediction has appeared fueled by the availability of rich feature representations, learned from raw data (Eigen D. a., 2015), (Laina, 2016), (Casser, 2019.). These approaches outperformed classical methods in terms of depth estimation accuracy (Karsch K. C., 2014), (Liu B. S., 2010).

Numerous methods used supervised learning to estimate depth from a single view (Wang X. D., 2015), (Ocal, 2020). Despite supervised learning receives wide fame with depth prediction, it needs costly depth sensors for the training process. Therefore, many methods turn to using unsupervised learning image-to-depth techniques. Unsupervised depth prediction models have shown to be more accurate and get better performance than sensor-supervised methods (Zhan, 2018.). Several consequent works result in good performance with the monocular setting (Yang $Z$. P., 2017), (Yin, 2018.). However, these methods are still incomplete because they didn't handle object movements in dynamic scenes. These methods lead to failure as they cannot explain object motion in complex dynamic scenes.

Camera Pose Estimation, Monocular Visual SLAM or Visual Odometry (VO) methods that include feature-based methods (Mur-Artal R. J., 2015), (Klein, 2008) and direct methods (Engel, 2017), (Forster, 2014) are considered as a key tracking method for motion estimation. However, these methods lack of accurate depth estimation and are unable to handle pure rotation motions. CNN-SLAM (Tateno, 2017) and ORB-SLAM2 (Mur-Artal R. a., 2017) solved monocular SLAM limitations by using deep neural networks to improve the scene depth. Nevertheless, these methods fail to give good performance in a dynamic scene. Casser at el (Casser, 2019.) have proposed a novel technique to solve the monocular depth and ego-motion problem by explicitly modeling 3D motions of moving objects, together with camera ego-motion, and adapts to new environments by learning with an online refinement of multiple frames.

3D Reconstruction, the 3D reconstruction approaches are used for several domains. Table 1 summaries the main characteristics of the most relevant publications to our proposed framework Nowadays, most state-of-the-art research used neural network techniques to reconstruct 3D objects from a single RGB image. Audrius et al. (Kulikajevas, 2019) applied hybrid neural network architecture to reconstruct polygonal meshes from a single depth frame using RGB-D sensors 
125

126

127

128

129

130

131

132

133

134

135

136

137

138

139

140

141

142

143

144

145

146

147

148

149

150

151

152

153

154

155

156

157

158

159

160

161

162

163

164

165

166

167

168

169 devices. Despite the RGB-D sensor still being capable to use. It is a lack to capture reliable depth images from the outdoor scene. Also, approach presented in (Li, 2019) used generation adversarial networks (GANs) to reconstruct a 3D object. GANs can generate 3D models by sampling from uniform noise distribution and get a significant performance. However, the authors succeeded to generate a 3D model using the GANs network but it is not tested to reconstruct full 3D scene (static or dynamic). Another approach focused on a single model in the medical domain is (Widya, 2019). They illustrated a 3D reconstruction technique for the whole stomach. Structure-from-Motion (SfM) with a monocular endoscope is used. The authors study the combined effect of chromoendoscopy and color channel selection on SfM to increase the number of feature points and obtain more reliable reconstruction quality and completeness.

Scene reconstruction (static or dynamic) from video frames is one of the most important problems in computer vision field. This is because not only needs to deal with the camera pose, but also the object motions. Most of the RGB-D cameras have the natural advantage of reconstructing dense models, and many exciting fusion schemes are proposed in this domain (J. Lee, 2016 ) (Z. Yan, 2017). However, the received depth image from the depth camera contains noise or even outliers due to lighting conditions and spatially variant materials of the objects. (Wang J. H., 2018) improved this limitation by combing learning depth from RGB-D datasets with monocular SLAM and frame-wise point cloud fusion to build a dense 3D model of the scene. They can reconstruct smooth and surface-clear on various examples with a dedicated point cloud fusion scheme.

The 3D reconstruction of a dynamic scene is more challenging than the static scene. This is because it does not only need to deal with the camera pose, but it also deals with the object motion. In the past few years, there was a great interest to solve $3 \mathrm{D}$ scene reconstruction with moving objects using single or multiple Monocular camera RGB frames. Xingbin et al. (Yang X. L., 2020), presented a real-time monocular $3 \mathrm{D}$ reconstruction system for mobile phone which used online incremental mesh generation for augmented reality application. For the $3 \mathrm{D}$ reconstruction process, they performed monocular depth estimation with a multi-view semi-global matching method followed by a depth refinement post-processing. Because the dynamic objects such as walking pedestrians or moving objects not support by multi-view geometry prerequisites, the authors deal with this problem by update the algorithm to remove the dynamic objects from the reconstruction mesh.

On the other hand, several applications focused on 3D reconstruction for a specific category of moving objects such as full or part of the human body. (Shimada, 2020) illustrated markless 3D human motion capture from monocular videos. They concentrated on challenging 3D motion observed, such as foot sliding, foot-floor penetration, and unnatural body leaning. Also, (Peng, 2020) proposed $3 \mathrm{D}$ hand mesh reconstruction from a single RGB image. The authors relied on the graph convolution neural network $(\mathrm{GCN})$ with two modules; hand localization and mask generation, to capture geometric details of 3D hand shape.

On the level of a dynamic outdoor domain, several domains are interested in predicting future $3 \mathrm{~d}$ scenes from existing ones. (Lu, 2020) and (Weng, 2020. )using neural networks such as LSTM and GRU to generate a full 3D point cloud from outdoor LiDAR datasets. The main idea is to use the motion-based neural network that integrates motion features between two consecutive point clouds.

Further, $(\mathrm{Ku}, 2019$.) introduced a monocular 3D object detection method that leverages proposals and shapes reconstruction. This method depends on three main processes; geometric priors, shape reconstruction, and depth prediction. The feature map is produced by image crop of the object and

Peer] Comput. Sci. reviewing PDF | (CS-2020:12:55956:2:1:NEW 6 Apr 2021) 
170 global context as input for the network. The orientation is predicted to estimate a proposal. 171 Moreover, the point-cloud is generated and transformed into the global frame.

172 (Kumar S. D., 2019) illustrates a technique to build 3D reconstruction of a complex dynamic scene

173 using two frames by applying super-pixel over-segmentation to the image. A generically dynamic

174 (hence non-rigid) scene with a piecewise planar and rigid approximation are presented. Moreover,

175 they reduced the reconstruction problem to a "3D jigsaw puzzle" which takes pieces from an

176 unorganized "soup of super-pixels".

177 This work aims to create an accurate 3D scene model that recognizes every moving object from

178 monocular RGB video frames without sensor data. The 3D reconstruction process learns

179 information (depth map, camera pose, and object motion) from the current RGB frame, previous

180 frame, and keyframes to detect changes during the object's motion. The 3D point cloud is

181 continuously improved during this process by adding or removing some points according to a

182 certain certainty filter. Studying moving objects from video frames solve several problems such as

183 objects occlusion and collision in a dynamic outdoor environment such as augmented reality.

184

185

\section{The Proposed Framework}

186

In this section, a novel framework for 3D dynamic scene reconstruction is proposed. This framework consists of two stages/modules. In the first stage, unsupervised learning is used to predict scene depth, camera pose, and object motion for a complex dynamic scene. Second, during the online refinement process, the previous information is used to create a point cloud for each single frame. Figure 2 explains the framework stages starting from video frames till the generation of the full scene point cloud.

\section{The online refinement process}

This process was inspired by (Casser, 2019.). The advantage of this approach is not only predicting scene depth and camera pose, but also considering the motion of objects in a dynamic scene. Moreover, the framework gives better performance in outdoors than indoor scenes. The purpose of this approach is to represent the learnt geometric structure in the learning process by modeling the scene and the individual objects. From monocular videos, the camera ego-motion and object motions are recognized. Furthermore, the online refinement method is used to adapt to learn on the fly to unknown domains. The depth function is a full convolution encoder-decoder architecture based on residual network (ResNet18) architecture (He, 2016). It begins with pre-trained weights on ImageNet (Deng, 2009), which produces a dense depth map from a single RGB frame. Moreover, the camera-motion neural network takes a sequence of two RGB images as input and generates an SE3 transform between the frames.

For object motion, the segmentation mask is used between two RGB images for every individual object. RCNN architecture with initialized pre-trained on the COCO dataset (Lin, 2014). In this stage, the framework predicts the transformation vector (Translation and rotation) for every single object in 3D space, which creates the detected object appearance in the respective target frame. The static background is generated by a single warp based on ego-motion.

\section{Point Cloud Fusion Phase}


215 The estimated RGB depth, camera pose, and object motion learnt in the previous phase is 216 transformed to the frame-wise point cloud and later an accurate 3D model is reconstructed for the 217 dynamic environment. The predicted 6-dimensional transformation vector (ego-motion) is used to 218 convert the estimated depth into a unified coordinate space and then fused into an accumulated

219

220

221

222

223

224

225

226

227

228

229

230

231

232

233

234

235

236

237

238

239

240

241

242

243

244

245

246

247

248

249

250

251

252

253

254 global model. The global model is a list of 3D points with correlating characteristics. For each frame, the per-frame point cloud is generated. The stability of each pixel in the current frame is checked with all co-visible keyframes. If corresponding points are found at any co-visible keyframe, the most stable point is merged with the new estimated point using a weighted average insertion. If not found, the estimated point is added to the global model as an unstable point. The global model is cleaned up overtime to remove outliers due to stability and temporal constraints. The selection process of co-visible keyframes depends on the total number of video frames and positions of co-visible keyframes. Many experiments have been conducted to select a suitable number of co-visible keyframes with their corresponding positions. As a result, it is found that five co-visible keyframes distributed over 20 frames gives an acceptable accuracy.

\section{Proposed Monocular 3D Model Reconstruction}

In this section, the proposed framework for 3D model reconstruction in a complex dynamic scene using monocular video frames will be illustrated. The proposed framework is divided into two main phases; unsupervised learning techniques phase for depth, camera pose and object motion, and point cloud frame-wise phase for a sequence of monocular video frames.

\section{Learning Techniques Phase for Frame Depth, Camera Pose, and Object Motion}

The sequence of three RGB frames from monocular camera is used for the learning process $\left(I_{1}, I_{2}, I_{3}\right)$. The camera intrinsic matrix is defined as $K \in R^{3 \times 3}$. Depth prediction uses a single frame to produce a depth map. The depth map $D_{i}=\theta\left(I_{i}\right)$ is generated by a fully convolutional encoder-decoder architecture $\theta: R^{H \times W \times 3} \rightarrow R^{H \times W}$ (ResNet 18). In contrast, ego-motion network $\psi E: R^{2 x H x w x 3} \rightarrow R^{6}$ takes sequence of two frames and produces a SE3 transform vector (Translation and Rotation) between frames. Different warping operation in one frame is used to adjacent one in sequence. It allows predicting how the scene seems like with a different camera viewpoint. Using different frame warping operator $\varnothing\left(I_{i}, D_{j}, E_{i \rightarrow j}\right) \rightarrow \hat{I}_{i \rightarrow j}$, where $\hat{I}_{i \rightarrow j}$ is the reconstructed $j$-th image. This approach able to change any source RGBimage $I_{i}$ into $I_{j}$ given corresponding depth estimate $D_{j}$ and an ego-motion estimate $E_{i \rightarrow j}$.

In practice, $\varnothing$ performs the warping by reading from transformed frame pixel coordinates. The projected coordinates are calculated by setting $\hat{I}_{i \rightarrow j}^{x, y}=\hat{I} \hat{i \rightarrow j}$ where $[\hat{x}, \hat{y}, 1]^{T}=K E_{i \rightarrow j}\left(D_{j}^{x, y} \cdot K^{-1}\right.$ $\left.[x, y, 1]^{T}\right)$. The reconstruction loss for this approach is calculated as mentioned in (Casser, 2019.).

The object motion model $\psi M$ is used to predict the motion of individual objects in 3D space. Similar to ego-motion architecture, it used a sequence of two RGB frames. The object motion 
applied a segmentation mask (RCNN architecture) for individual objects into the dynamic scene. The transformation vector per object is learned, which creates the detected object appearance in the respective target form. According to the ego-motion model, the static background is generated and then all segmented objects are added by their appearance. The advantage of this approach is not only modeling objects in 3D space, but also learning their motion on the fly.

\section{D Model Reconstruction with Point Cloud Fusion}

After depth, camera pose and object motion are predicted in the previous stage. It is time to reconstruct a full 3D scene model using point cloud fusion. The point cloud generation is inspired by ORB-SLAM2 (Mur-Artal R. a., 2017), (Wang J. H., 2018). (Wang J. H., 2018) used point cloud fusion to generate $3 \mathrm{D}$ model reconstruction suitable for augmented reality applications. The advantage of this approach is that it is easy to apply and convenient for realtime applications. The limitation of this approach is being limited to a static environment and is not tested in a dynamic environment. The proposed framework alleviates this limitation to consider objects motion' in dynamic environments. The per-frame point cloud is reconstructed by estimating depth $\boldsymbol{D}_{\boldsymbol{i}}$ for a single frame, the final Motion $\boldsymbol{E}_{\boldsymbol{m}}^{\boldsymbol{F}}$ that is a combination of individual moving objects $\psi M_{o}$ and Camera motion $\psi E_{\boldsymbol{i} \rightarrow \boldsymbol{j}}$. Once the intrinsic camera calibration matrix $K$ is given, the per-frame point cloud is calculated as following:

$$
p_{i}=\left(E_{m}^{F}\right)^{-1} \pi\left(u, D_{i}\right)
$$

Where $u$ denote as homogeneous representation of a pixel $u=(x, y, 1)^{T}$ and $\pi(u)$ is the back projection from image to camera coordinate, $\pi\left(u, D_{i}\right)=K^{-1} D_{i} u$.

\section{Point Association}

The system holds a set of co-visible of keyframe $k_{1}, k_{2} \ldots k_{n} \in K F$ selected according to the length of monocular video frames on the online refinement process. The visibility of pixels is checked by mapping each pixel of the current frame with all co-visible keyframes.

$$
u^{k}=f\left(K\left(E_{m}^{F}\right)^{k} \pi\left(u^{i}\right)\right)
$$

where $\boldsymbol{f}(\boldsymbol{x})=\left(\frac{\boldsymbol{x}}{\boldsymbol{z}}, \frac{\boldsymbol{z}}{\mathbf{z}}\right)^{\boldsymbol{T}}$, also, maintain such a mapping from every keyframe pixel to its corresponding 3D point $M: p_{i} \rightarrow u^{i} \rightarrow u^{k} \rightarrow P$. where $\mathrm{P}$ is a global model.

To create a smooth 3D point cloud and filter out the noise from the generated points, the probabilistic filter is used. Each 3D point in global model $\mathrm{P}$ is represented by $p_{i}^{n}$, and the confidence counter $C_{c}$ is defined as how often the 3D point is observed in co-visible 
293

294

295

296

297

298

299

300

301

302

303

304

305

306

307

308

309

310

311

312

313

314

315

316

317

318

319

320

321

322

323

324

325

326

327

328

329

330

keyframes. The $C_{c}$ determines if $3 \mathrm{D}$ point evolves from unstable to stable state. Our weighted average is calculated by applying a Gaussian weight to the current depth measurement as $w_{A}$ $=\boldsymbol{e}^{-\gamma^{2} / \sigma^{2}}$ where $\gamma$ is the normalized radial distance of $D_{i}$ from the center of the camera, and $\sigma=0.6$. The new observation available in the latest frame $\boldsymbol{i}$ according to the following equations:

$$
p_{i}^{n}=\left(w_{A} p_{i}+w^{0}\left(E_{m}^{F}\right)^{-1} \pi\left(u^{i}\right)\right) /\left(w_{A}+w^{0}\right)
$$

$$
C_{C}^{n}=\left(w_{A} C_{c}+w^{0}\left\|\left(E_{m}^{F}\right)^{-1} \pi\left(u^{i}\right)-p_{i}\right\|\right) /\left(w_{A}+w^{0}\right)
$$

$$
w_{A}^{n}=\min \left(w_{A}+w^{0}, W_{\varepsilon}\right)
$$

Where $p_{i}^{n}$ means the newly updated point, $w^{0}$ is a constant equal to 1 and $W_{\varepsilon}$ is the truncation threshold equal to 100 (Wang J. H., 2018).

Figure 3 represents the pseudo code of 3D model reconstruction process. The point association start from line 8 to 25 ; in which the stability of each pixel in the current frame is checked with all covisible keyframes. If the corresponding points are found, the point is updated and set as stable according to the value of its corresponding confidence counter. If it is not found, the estimated point is added to the global model as an unstable point and inserted to point map as new seed. Probabilistic noise filter is applied at line 27 to maintain only stable points.

\section{Proposed Monocular 3D Model Reconstruction Implementation}

\section{Dataset and implementation details}

The proposed framework was evaluated by using KITTI dataset (Geiger, 2013). The KITTI dataset is the most recent dataset used in different applications because it contains different objects, and it is considered a complex dynamic environment. The KITTI dataset has LIDAR sensor readings for evaluation only. It is used to evaluate predicted depth and egomotion. Moreover, the KITTI 3D point cloud is used as ground truth to evaluate the proposed 3D model reconstruction model.

The number of points per scan is not constant, on average each frame has a size of $\sim 1.9$ MB which corresponds to $\sim 120,0003 \mathrm{D}$ points.

The proposed framework is divided into two modules/phases as mentioned in section 3 . The first module is responsible for predicting depth and ego-motion using unsupervised learning in a dynamic scene. The code implemented using TensorFlow, the actual size of input images is $1224 \times 368$, the images are resized to $416 \times 128$, the same setting which described in (Casser, 2019.) is used such as learning rate 0.0002 , L1 reconstruction weight 0.85 , SSIM weight 0.15 , smoothing weight 0.04 , object motion constraint weight 0.0005 , 
and batch size 4. The dataset is divided into training, validation, and testing (30542 monocular triplets for training, 3358 for validation, and 698 for testing). The framework has been executed on high-performance computing (HPC), PowerEdge Dell R740 (2x intel Xeon Gold 6248 2.5G) with Tesla V100 GPU. The second module generates a 3D point cloud to reconstruct a dynamic scene. This module is implemented in a framework of TensorFlow using Python, OpenGL, and open3D. During the online refinement process, 20 frames are selected to generate a 3D point cloud for each frame and finally integrated into one 3D model of the scene.

\section{Evaluation Metrics}

The evaluation process used three methods:

1- Localization Accuracy Error $L_{E}$, FPE (False positive error), and FNE (False Negative error) which were proposed by Refs (Hafiz, 2015).

Localization Accuracy, $L_{E}$ is defined as the amount of deviation of the detected point from a ground truth point position. Let ground truth data set denoted by $G_{T}$, ground truth point denoted by $p_{g} \in G_{T}, P_{p}$ is predicted points by the proposed technique. $N_{G}$ is the number of points in $G_{T}, N_{p}$ is the number of points in $P_{p}$, and $C_{r}\left(p_{g}\right)$ is geodesic distance over a region, which is centered by point $\mathrm{g}$ and has radius of $\mathrm{r}$. The data which is contained in $C_{r}\left(p_{g}\right)$ can be defined as:

$$
C_{r}\left(p_{g}\right)=\left\{p_{c} \in P_{p} \mid \operatorname{Min}\left(\operatorname{dis}\left(p_{g}, p_{c}\right)\right)<r\right\}
$$

where $\operatorname{dis}\left(p_{g}, p_{c}\right)$ is the Euclidian distance between the two points $p_{g}$ and $p_{c}$, and $r$ is the Maximum correspondence points-pair distance which controls the localization error. $p_{c}$ is considered to be correctly detected if there exists a detected point $p_{c} \in P_{p}$ $\cap C_{r}\left(p_{g}\right)$ such that $p_{c}$ is the minimum distance between the points $p_{g}$ and $p_{c}$. The $L_{E}$ defined as follows:

$$
L_{E}=\sqrt{\frac{1}{N_{C}}} \sum_{j}^{N} C_{d i s}\left(p_{g j}, p_{c j}\right)
$$

where $N_{C}$ is the number of correctly detected points in $G_{T}$.

The FNE at localization error tolerance $r$ is defined as:

$$
\operatorname{FNE}(r)=1-\frac{N_{C}}{N_{G}}
$$

The FPE at localization error tolerance $r$ is defined as: 
365

366

367

368

369

370

371

372

373

374

375

376

377

378

379

380

381

382

383

384

385

386

387

388

389

390

391

392

393

394

395

396

397

398

The number of false positives is normalized with the number of all true negatives, where $N_{F}$ is the number of false positives, and yields to

$$
N_{F}=N_{p}-N_{C}
$$

2- Registration 3D point cloud between the output of the Velodyne laser scanner (ground truth) and the proposed technique which generates a 3D point cloud from the predicted depth and predicted ego-motion. Global registration (Zhou, 2016) and Iterative Closest Point ICP point-to-point (Rusinkiewicz, 2001.) (Paul, 1992) are used. Moreover, evaluate the registration by calculating Fitness function which is used to measure the overlapping area (the number of inlier correspondences/number of points in ground truth). The Higher value of fitness is better. While for the Root Mean Square Error RMSE of all correspondences in range of $r$, the lower is better.

3- Accuracy is reported using mean relative error (MRE). Which defined as

$$
M R E=\frac{1}{P} \sum_{i=1}^{\mathrm{P}} \frac{\left|z_{g t}^{i}-z_{e s t}^{i}\right|}{z_{g t}^{i}}
$$

Let $z_{g t}^{i}, z_{e s t}^{i}$ are the ground-truth depth and the estimated depth respectively with $P$ as the total number of $3 \mathrm{D}$ point Cloud. MRE is state of the art metric used to compare the proposed framework with several monocular dynamic reconstruction methods.

Another used metric is Chamfer Distance (CD) between the ground truth $P_{g t} \in R^{N \times 3}$ point cloud and the estimated point cloud $P_{e s t} \in R^{N \times 3}$. Chamfer Distance (Lu, 2020) is a regularly used metric to measure the similarity between two-point clouds, which is define as:

$$
C D=\frac{1}{N} \sum_{x^{\wedge} \sum_{i} \in P_{e s t}} \min _{x}{ }_{x} \in P_{g t}\left|x^{\wedge i}-x^{j}\right|+\frac{1}{N} \sum_{x^{j} \in P_{g t}} \min _{x^{\wedge}{ }^{i} \in P_{e s t}}\left|x^{\wedge i}-x^{j}\right|
$$

\section{Experimental Results}

Localization Accuracy

In this section, the proposed framework has been evaluated using different techniques. At first, localization error $L_{E}$, FNE, and FPE using different localization error tolerance $r$ between ground truth and predicted 3D point cloud is calculated. Figure 4 illustrates the average of localized error, FNE, and FPE with $r$ in range 0.04 to 0.16 for 20 frames, and the output of the online refinement process. Form Figure 4, it is found that the proposed framework is succeeded in finding points close to ground-truth 
points with low localization error. Moreover, decreasing in FNE indicates that the framework catches the nearest points with a low localization error, while a rapid drop in FPE means that the framework does not return excessive interest points.

\section{Point Cloud Registration}

The second approach using 3D point cloud registration between ground-truth and predicted points is used to check the accuracy of 3D reconstruction. Figure 5 shows a selected frame from a sequence of 20 frames registered with ground-truth with two different points of view. The number of predicted 3D point cloud depends on the number of $2 \mathrm{D}$ pixels of the RGB frame. In our case, the input frame size is $416 \times 128$ (in range of 50000 points), counter to the $3 \mathrm{D}$ point cloud of ground truth that collected from the Velodyne laser scanner is in the average of 120000 points. As shown in Figure 5, the range and density of the predicted 3D point cloud are less than the ground truth. Therefore, the predicted 3D point cloud is closed to the ground-truth in the selected area.

To evaluate the registration between ground-truth and predicted 3D point cloud, we used the state of the art algorithms such as Global registration (Zhou, 2016) and ICP point-topoint (Rusinkiewicz, 2001.) (Paul, 1992). Figures 6 and 7 illustrate the average of RMSE and fitness of registration for 20 frames on online refinement process using Global registration and ICP point-to-point with different threshold.

As shown in figures 6 and 7, ICP point-to-point registration gets the lowest RMSE and higher fitness between ground truth and predicted 3D point cloud. This, is because the ICP point to point technique usually runs until convergence or reaches a maximum number of iterations (we used the default iteration 30). This indicates that the proposed framework succeeds to cover large number of an overlapping areas with a small mean square error.

Figure 8 gives more details about ICP point-to-point registration during 20 frames with an acceptable RMSE and stander deviation with the increasing of $r$ value.

Figure 9 illustrates the 3D point cloud after mapping on RGB frames selected from different videos. Figure 9 (b) is a referee to the ground-truth point, and Figure 9 (c) to the predicted point from our framework. The performance of the proposed framework is compared with the state-of-the-art methods (Kumar S. Y., 2017), which reported that MRE on KITTI dataset and with several monocular dynamic reconstruction methods, such as the Block Matrix Method (BMM) (Dai, 2014), Point Trajectory Approach (PTA) (Akhter, 2010), and Low-rank Reconstruction (GBLR) (Fragkiadaki, 2014) , Depth Transfer (DT) (Karsch K. C., 2012.), and (DMDE) (Ranftl, 2016). Note that we used the reported result in (Kumar S. Y., 2017) as its implementation is not available publicly. 
439

440

441

442

443

444

445

446

447

448

449

450

451

452

453

454

455

456

457

458

459

460

461

462

463

464

465

466

467

468

469

470

471

472

473

474

475

476

477

Figure 10 shows that the proposed framework delivers consistently superior reconstruction accuracy on the KITTI dataset. Using unsupervised learning to predict scene depth and camera pose is a strong point of the proposed framework for generating an accurate $3 \mathrm{D}$ model reconstruction. Table 2 shows the improvement percentages between the proposed framework and the state-of-the-art methods.

\section{Chamfer Distance}

We calculate the Chamfer Distance (CD) between the point cloud and ground truth on the KITTI dataset and compare it with state-of-the-art modules proposed in ( $\mathrm{Lu}, 2020)$. The main idea of this metric is to predict future frames given the past point cloud sequence based on a motion-based neural network named MoNet. Two neural networks are used to predict scene point-cloud LSTM and GRU.

The main idea of these methods using point-cloud as an input for neural networks to estimate future frames. The MoNet integrates motion features into the prediction pipeline and combines them with content features. In this metric, we used the average CD for 5 frames to match with the module (Lu, 2020). As shown in Table 3, the CD of our framework is slightly better than Mon (LSTM) and Mon (GRU) methods.

Finally, the experimental results show how the proposed framework achieves an accurate 3D reconstructed point-cloud model from monocular RGB video frames without using expensive sensors. Several evaluation metrics are measured, such as Localization error, RMSE, and Fitness between ground truth and predicted point-cloud. Finally, the experimental results show how the proposed framework achieves an accurate 3D reconstructed point-cloud model 1 from monocular RGB video frames without using expensive sensors. Several evaluation metrics are measured, such as Localization error, RMSE, and Fitness between ground truth and predicted point-cloud. Moreover, we achieved 46\% improvement in MRE error compared with the state-of-the-art method DJP. Besides, $11 \%$ and 14\% improvement using chamfer distances metric compared with MonNet (GRU) and MoNet (LSTM) respectively.

\section{The limitations:}

The success of the presented framework depends on the accuracy of learning parameters such as depth map, camera pose, and object motion. In the case of the learning parameters are not processed accurately in phase one, the $3 \mathrm{D}$ reconstruction will fail. The other major limitation is the overall execution time. Because the generation of a 3D point cloud depends on accumulative matching between the current frame and a group of keyframes, this process takes up to 15 minutes. Moreover, the proposed framework had reconstructed a point cloud from a sequence of 20 RGB video frames which is considered a short sequence. However, this limitation could be overcome by using parallel programming to handle the most time-consuming part of the 3D point cloud reconstruction as mentioned in the future work section.

\section{Conclusion and Future Work}


478 This paper proposes a novel framework for 3D model reconstruction from monocular video frames 479 for a dynamic environment. The framework didn't use any sensor data, which is costly and 480 sometimes noisy. The results showed that the proposed framework is capable of generating smooth 481 and accurate $3 \mathrm{D}$ point-cloud for a dynamic environment using cumulative information of a 482 sequence of RGB video frames. Different evaluation metrics are used such as Localization error 483 and RMSE with average values of 0.05 and 0.067 respectively between ground truth and predicted point-cloud. Moreover, the increase in fitness value indicates that the proposed framework succeeded to cover a large number of overlapping areas with a small mean square error.

Furthermore, a comparison between the proposed framework and state-of-the-art method using MRE compared with the DJP technique and Chamfer Distance compared with two MoNet techniques with an improvement of $46 \%, 11 \%$, and $14 \%$ respectively. In the future, we will be concerned with improving the overall execution time to make it able to deal with real-time applications such as augmented reality by applying several optimization techniques using state-ofthe-art GPU and CUDA platforms. In addition to, test 3D model reconstruction over long sequences of RGB frames.

493

494

495

496

497

498

499

500

501

502

503

504

505

506

507

508

509

510

511

512

513

514

\section{References}

Akhter, I. Y. (2010). Trajectory space: A dual representation for nonrigid structure from motion. . IEEE Transactions on Pattern Analysis and Machine Intelligence, 33(7), 1442-1456.

Casser, V. S. ( 2019.). Depth prediction without the sensors: Leveraging structure for unsupervised learning from monocular videos. In Proceedings of the AAAI Conference on Artificial Intelligence, vol. 33, (pp. 8001-8008.).

Chen, K. Y.-K.-M. (2015). 3D indoor scene modeling from RGB-D data: a survey. Computational Visual Media 1, no. 4 , (pp. 267-278.).

Dai, Y. H. (2014). A simple prior-free method for non-rigid structure-from-motion factorization. International Journal of Computer Vision, 107( 2 ), 101-122.

Davison, A. a. (2011). KinectFusion: real-time 3D reconstruction and interaction using a moving depth camera. In Proceedings of the 24th Annual ACM Symposium on User Interface Software and Technology (UIST 2011), (pp. 559-568).

Deng, J. W.-J.-F. (2009). Imagenet: A large-scale hierarchical image database. In 2009 IEEE conference on computer vision and pattern recognition (pp. 248-255.). IEEE.

Eigen, D. a. (2015). Predicting depth, surface normals and semantic labels with a common multi-scale convolutional architecture. In Proceedings of the IEEE international conference on computer vision, (pp. 2650-2658).

Eigen, D. C. (2014). Depth map prediction from a single image using a multi-scale deep network. In Advances in neural information processing systems, 2366-2374.

Engel, J. V. (2017). Direct sparse odometry. IEEE transactions on pattern analysis and machine intelligence 40, no. 3, (pp. 611-625).

Peer) Comput. Sci. reviewing PDF | (CS-2020:12:55956:2:1:NEW 6 Apr 2021) 
515 Findley, M. J. (2017). Locus of control and academic achievement: a literature review. Journal of $516 \quad$ personality and social psychology 44, no. 2 (1983): 419.

517 Forster, C. M. (2014). SVO: Fast semi-direct monocular visual odometry. . In 2014 IEEE international $518 \quad$ conference on robotics and automation (ICRA) (pp. 15-22). IEEE.

519 Fragkiadaki, K. M. (2014). Grouping-based low-rank trajectory completion and 3D reconstruction. . In

520 Advances in Neural Information Processing Systems, 55-63.

521

Furukawa, Y. a. (2009). Accurate, dense, and robust multiview stereopsis. IEEE transactions on pattern

522 analysis and machine intelligence 32, no. 8, (pp. 1362-1376.).

523

Geiger, A. P. (2013). Vision meets robotics: The kitti dataset. The International Journal of Robotics

524

525

526

Hafiz, D. A. (2015). Interest point detection in 3D point cloud data using 3D Sobel-Harris operator.

527

528

Hassan, M. A. (2017). 3D distance measurement accuracy on low-cost stereo camera. Science International Journal of Pattern Recognition and Artificial Intelligence , 29, no. 07, 1555014.

529 International 29, no. 3, (pp. 599-599).

He, K. X. (2016). Deep residual learning for image recognition. In Proceedings of the IEEE conference on 530 computer vision and pattern recognition, (pp. 770-778).

531

532

533

534

535

536

537

538

539

540

541

542

543

544

545

546

547

548

549

J. Lee, J. J. (2016 ). Low-cost depth camera pose tracking for mobile platforms. In Mixed and Augmented Reality(ISMAR-Adjunct) IEEE International Symposium (pp. 123-126). IEEE.

Karsch, K. C. (2012.). Depth extraction from video using non-parametric sampling.". In European Conference on Computer Vision. Berlin, Heidelberg.

Karsch, K. C. (2014). Depth transfer: Depth extraction from video using non-parametric sampling. IEEE transactions on pattern analysis and machine intelligence 36, no. 11 , (pp. 2144-2158).

Keller, M. D. (2013.). Real-time 3d reconstruction in dynamic scenes using point-based fusion. International Conference on 3D Vision-3DV (pp. 1-8). IEEE.

Klein, G. a. (2008). Improving the agility of keyframe-based SLAM. In European conference on computer vision (pp. 802-815). Berlin, Heidelberg: Springer.

Ku, J. A. ( 2019.). Monocular 3d object detection leveraging accurate proposals and shape reconstruction.". In Proceedings of the IEEE/CVF Conference on Computer Vision and Pattern Recognition (pp. pp. 11867-11876.). IEEE.

Kuhn, A. S. (2019). Plane completion and filtering for multi-view stereo reconstruction. Conference on Pattern Recognition (pp. 18-32). German : Springer.

Kulikajevas, A. R. (2019). Reconstruction of 3D object shape using hybrid modular neural network architecture trained on 3D models from ShapeNetCore dataset. Sensors 19, no. 7, 1553.

Kumar, S. D. (2019). Superpixel soup: Monocular dense 3d reconstruction of a complex dynamic scene. IEEE Transactions on Pattern Analysis and Machine Intelligence. 
550

551

552

553

554

555

556

557

558

559

560

561

562

563

564

565

566

567

568

569

570

571

572

573

574

575

576

577

578

579

580

581

582

583

584

Kumar, S. Y. (2017). Monocular dense $3 d$ reconstruction of a complex dynamic scene from two perspective frames. In Proceedings of the IEEE International Conference on Computer Vision.

Laina, I. C. (2016). Deeper depth prediction with fully convolutional residual networks. In 2016 Fourth international conference on 3D vision (3DV) (pp. 239-248). IEEE.

Li, H. Y. (2019). 3D model generation and reconstruction using conditional generative adversarial network. International Journal of Computational Intelligence Systems 12, no. 2 , 697-705.

Lin, T.-Y. M. (2014). Microsoft coco: Common objects in context. In European conference on computer vision (pp. 740-755). Cham: Springer.

Liu, B. S. (2010). Single image depth estimation from predicted semantic labels. In 2010 IEEE Computer Society Conference on Computer Vision and Pattern Recognition (pp. 1253-1260). IEEE.

Liu, F. C. (2015). Learning depth from single monocular images using deep convolutional neural fields. IEEE transactions on pattern analysis and machine intelligence 38, no. 10, 2024-2039.

Lu, F. G. (2020). MoNet: Motion-based Point Cloud Prediction Network. arXiv preprint arXiv:2011.10812.

Mur-Artal, R. a. (2017). Orb-slam2: An open-source slam system for monocular, stereo, and rgb-d cameras. IEEE Transactions on Robotics 33, no. 5, (pp. 1255-1262).

Mur-Artal, R. J. (2015). ORB-SLAM: a versatile and accurate monocular SLAM system. IEEE transactions on robotics 31, no. 5, (pp. 1147-1163).

Ocal, M. a. (2020). RealMonoDepth: Self-Supervised Monocular Depth Estimation for General Scenes. arXiv preprint arXiv:2004.06267 .

Paul, J. (1992). BESL and Neil MCKAY, A methode for registration of 3d shapes. IEEE Transactions on pattern analysis and machine intelligence, 14, (no. 2), 239-256.

Peng, H. C. (2020). 3D hand mesh reconstruction from a monocular RGB image. The Visual Computer 36, no. $10,2227-2239$.

Ranftl, R. V. (2016). Dense monocular depth estimation in complex dynamic scenes. In Proceedings of the IEEE conference on computer vision and pattern recognition.

Rusinkiewicz, S. a. (2001.). "Efficient variants of the ICP algorithm.". In Proceedings third international conference on 3-D digital imaging and modeling, IEEE, (pp. 145-152).

Shimada, S. G. ( 2020). Physcap: Physically plausible monocular 3d motion capture in real time. . ACM Transactions on Graphics (TOG), 39(6), pp.1-16.

Tateno, K. F. ( 2017). Cnn-slam: Real-time dense monocular slam with learned depth prediction. In Proceedings of the IEEE Conference on Computer Vision and Pattern Recognition, (pp. 62436252).

Ummenhofer, B. H. ( 2017.). Demon: Depth and motion network for learning monocular stereo. In Proceedings of the IEEE Conference on Computer Vision and Pattern Recognition, (pp. 50385047.).

Peer] Comput. Sci. reviewing PDF | (CS-2020:12:55956:2:1:NEW 6 Apr 2021) 
585

586

587

588

589

590

591

592

593

594

595

596

597

598

599

600

601

602

603

604

605

606

607

608

609

610
V. Pradeep, C. R. (2013). Monofusion: Real-time 3d reconstruction of small scenes with a single web camera. . In Mixed and Augmented Reality (ISMAR), IEEE International Symposium (pp. 83-88.). IEEE.

Wang, J. H. (2018). CNN-monofusion: online monocular dense reconstruction using learned depth from single view. IEEE international symposium on mixed and augmented reality adjunct (ISMARAdjunct) (pp. 57-62). IEEE.

Wang, X. D. (2015). Designing deep networks for surface normal estimation. In Proceedings of the IEEE Conference on Computer Vision and Pattern Recognition, (pp. 539-547).

Wang, Z. A. (2004). Image quality assessment: from error visibility to structural similarity. iEEE transactions on image processing 13, no. 4., (pp. 600-612).

Widya, A. R. (2019). Whole stomach 3d reconstruction and frame localization from monocular endoscope video. IEEE Journal of Translational Engineering in Health and Medicine, 1-10.

Yang, X. L. (2020). Mobile3DRecon: Real-time Monocular 3D Reconstruction on a Mobile Phone. IEEE Transactions on Visualization and Computer Graphics 26, no. 12 , 34.

Yang, Z. P. ( 2017). "Unsupervised learning of geometry with edge-aware depth-normal consistency." . arXiv preprint arXiv:1711.03665.

Yin, Z. a. (2018.). "Geonet: Unsupervised learning of dense depth, optical flow and camera pose." . In Proceedings of the IEEE Conference on Computer Vision and Pattern Recognition,, (pp. 19831992.).

Z. Yan, M. Y. (2017). Dense visual slam with probabilistic surfel map. IEEE transactions on visualization and computer graphics (pp. 2389-2398). IEEE.

Zhan, H. R. (2018.). "Unsupervised learning of monocular depth estimation and visual odometry with deep feature reconstruction.". In Proceedings of the IEEE Conference on Computer Vision and Pattern Recognition, (pp. 340-349.).

Zhou, Q.-Y. J. (2016). Fast global registration. n European Conference on Computer Vision, pp. 766-782. Springer. Cham.

611 


\section{Figure 1}

The proposed framework of 3D model reconstruction from monocular KITTI video images (Geiger, 2013)

KITTI dataset is under the Creative Commons Attribution-NonCommercial-ShareAlike 3.0

License. according to this link: http://www.cvlibs.net/datasets/kitti/
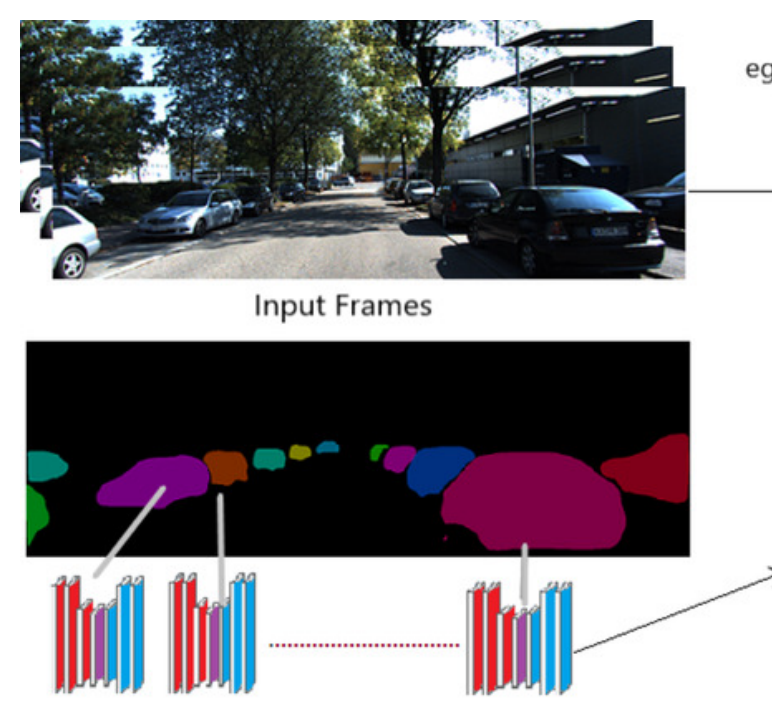

3D object Motion Estimator
ego-Motion Estimator

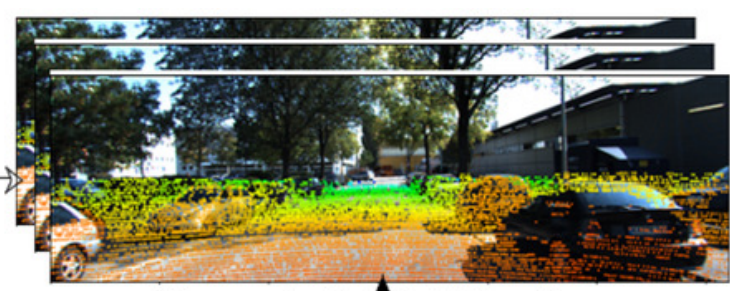

$3 \mathrm{D}$ reconstruction (Point Cloud)

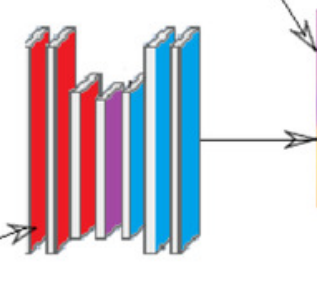

Depth Estimator

Online Refinment 
Figure 2

The Proposed Framework overview

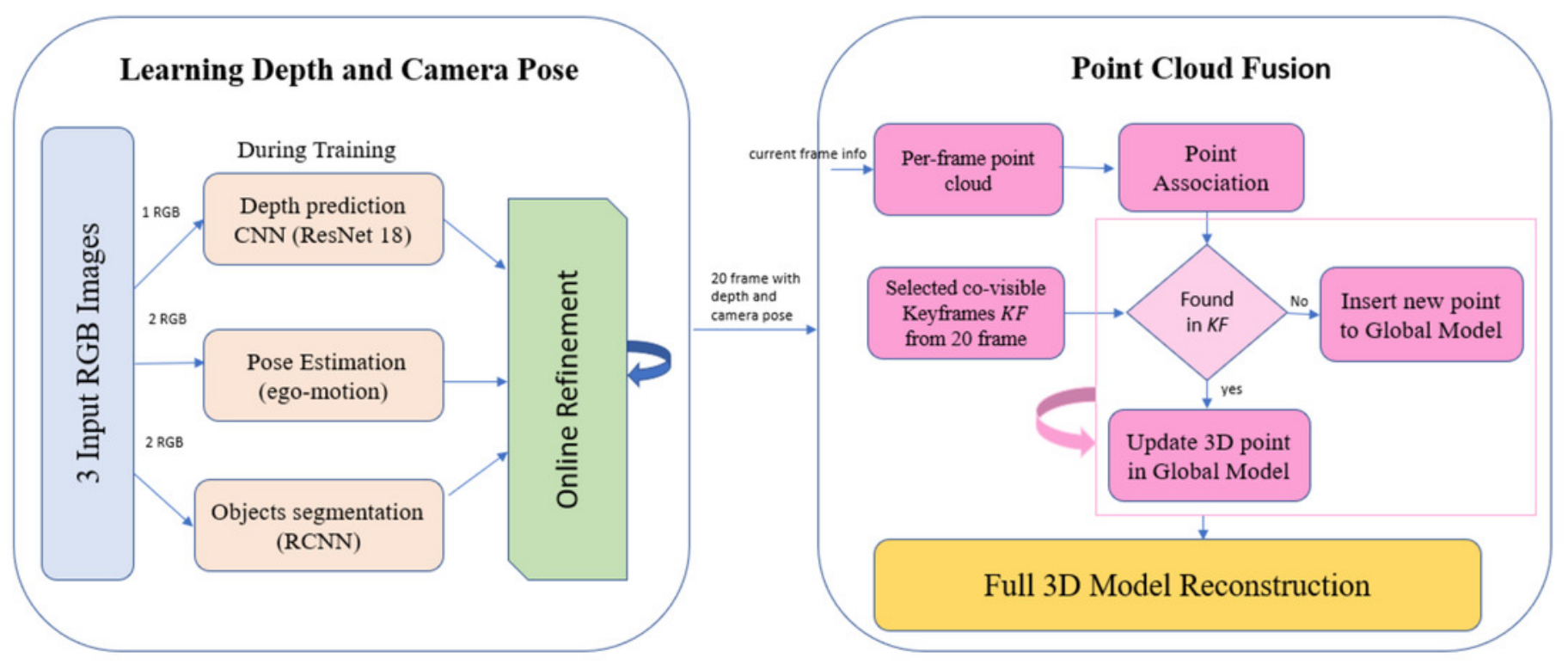


Figure 3

The pseudo code of 3D model reconstruction process. 


\section{Generate a 3D Reconstruction for dynamic scene}

Input: $\mathrm{P} \leftarrow$ global Model, hash map contains $3 \mathrm{D}$ point cloud, confidence counter, average weight, and point status (Stable, Unstable) with length $\mathrm{s}$

$\mathrm{M} \leftarrow$ point Map, mapping of $[\mathrm{x}, \mathrm{y}]$ and pointID for $u^{k}$ with length $\mathrm{s}$

$\mathrm{L} \leftarrow$ the $u^{k}$ dimension

$\mathrm{F} \leftarrow$ total number of frames

$\mathrm{KF} \leftarrow$ total number of Co-visible keyframe

1 for $i=1 \rightarrow F$ do

$2 \mid \%$ project current frame to world coordinates

$3 \mid p_{i}=\left(E_{m}^{F}\right)^{-1} \pi\left(u, D_{i}\right)$

4 | for $k=1 \rightarrow K F$ do

5 | | \% project world coordinates to co-visible keyframe

6 | $\mid p_{i} \rightarrow u^{i} \rightarrow u^{k}$

7||$\quad u^{k}=f\left(K\left(E_{m}^{F}\right)^{k} \pi\left(u^{i}\right)\right)$

8 | for $\mathrm{j}=l \rightarrow L$ do $\%$ point association

9|| if $\mathrm{j}$ in $\mathrm{M}$ :

$10|\quad| \quad \%$ point is visible

11

12

13 |

| $\%$ current frame-wise 3D point associated with the 3D global model $\%$ update point info

$$
\mid p_{i}^{n}=\left(w_{A} p_{i}+w^{0}\left(E_{m}^{F}\right)^{-1} \pi\left(u^{i}\right)\right) /\left(w_{A}+w^{0}\right)
$$

$$
\mid C_{C}^{n}=\left(w_{A} C_{c}+w^{0}\left\|\left(E_{m}^{F}\right)^{-1} \pi\left(u^{i}\right)-p_{i}\right\|\right) /\left(w_{A}+w^{0}\right)
$$

$$
\begin{aligned}
& \mid w_{A}^{n}=\min \left(w_{A}+w^{0}, W_{\varepsilon}\right) \\
& \mid \text { if } C_{C}^{n}<\text { stable_threshold }
\end{aligned}
$$

| \% point is stable

| else

| \% point unstable

| else

28

insert $p_{i}$ to $\mathrm{P} \%$ with all point info

| insert $\mathrm{n}$ to $\mathrm{M}$

Output: accumulated global model $\mathrm{P}$ for $3 \mathrm{D}$ reconstruction 
Figure 4

Localization error, FNE and FPE with different $r$ value

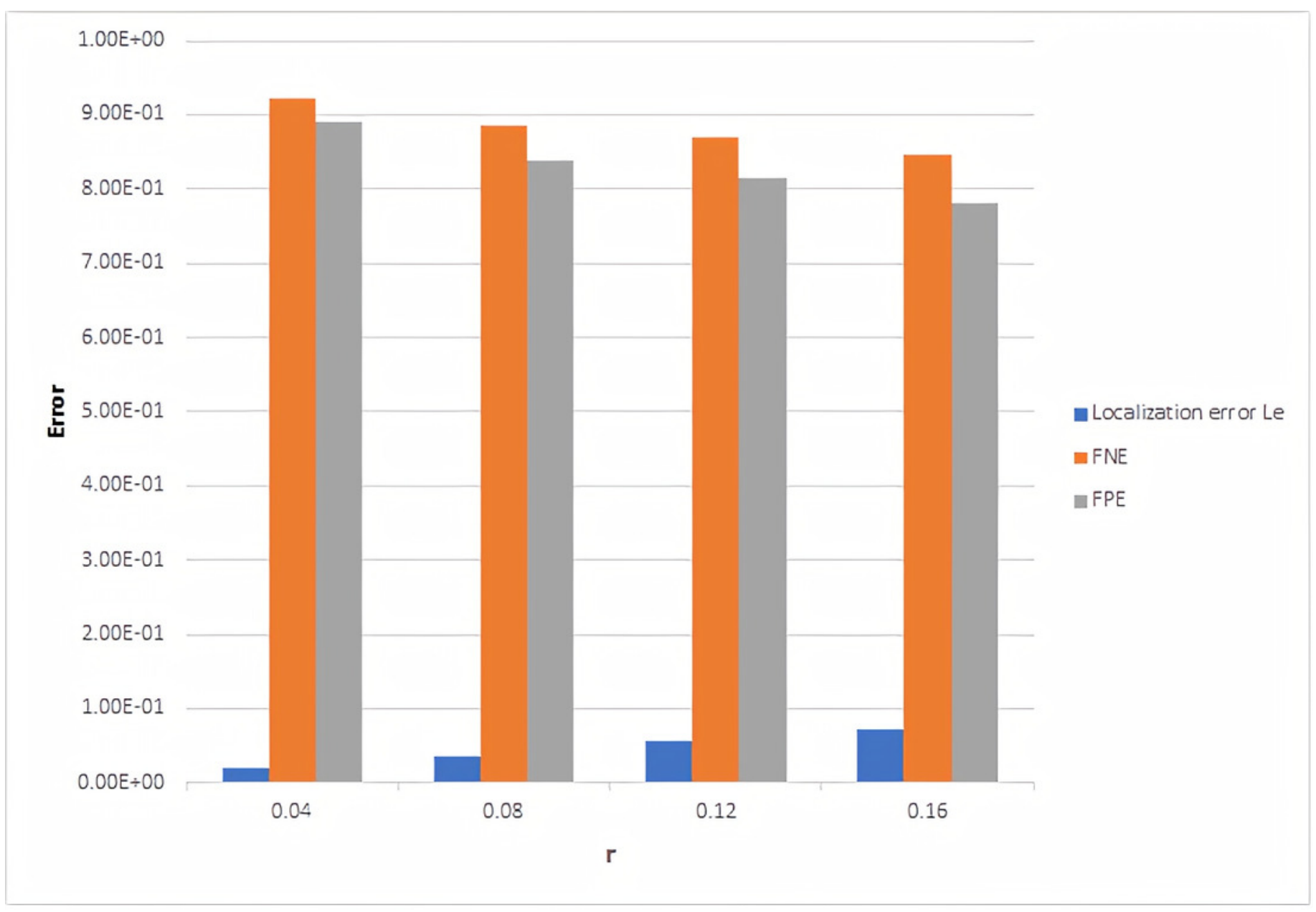


Figure 5

Registration between ground-truth(yellow) and predicted 3D point cloud (blue)

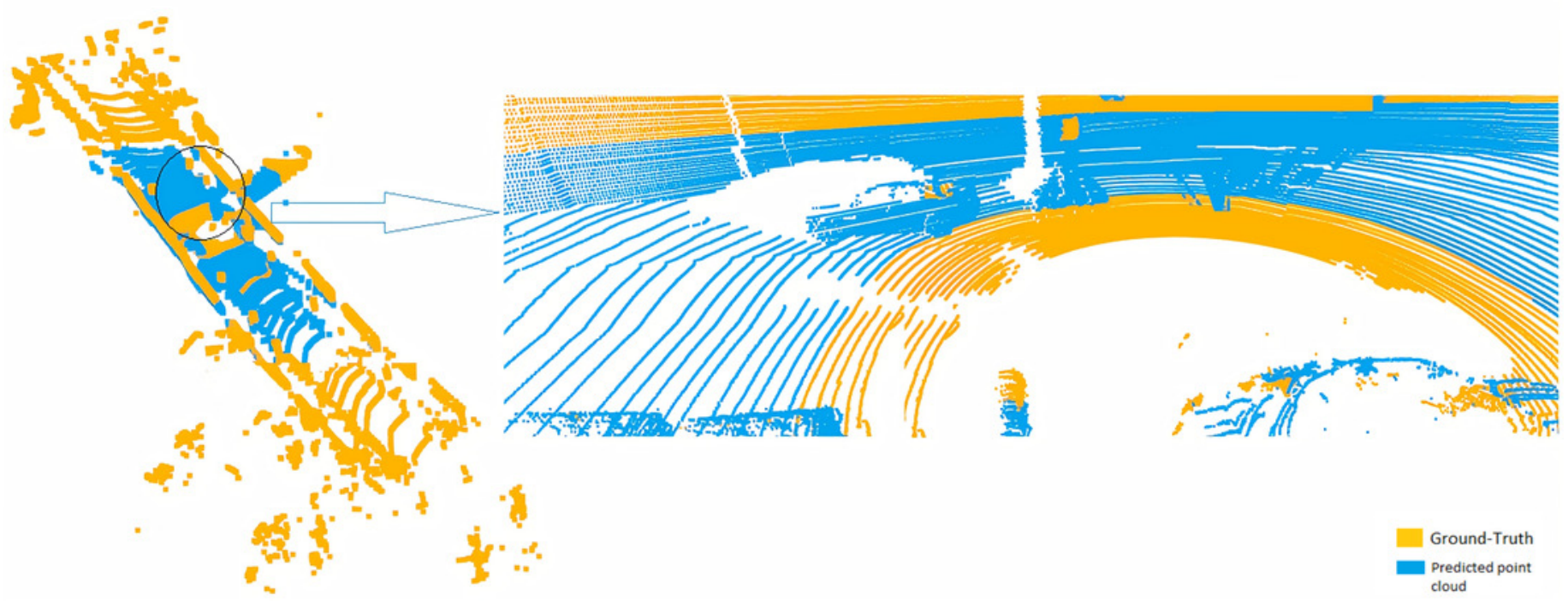


Figure 6

\section{Average RMSE for 20 frames}

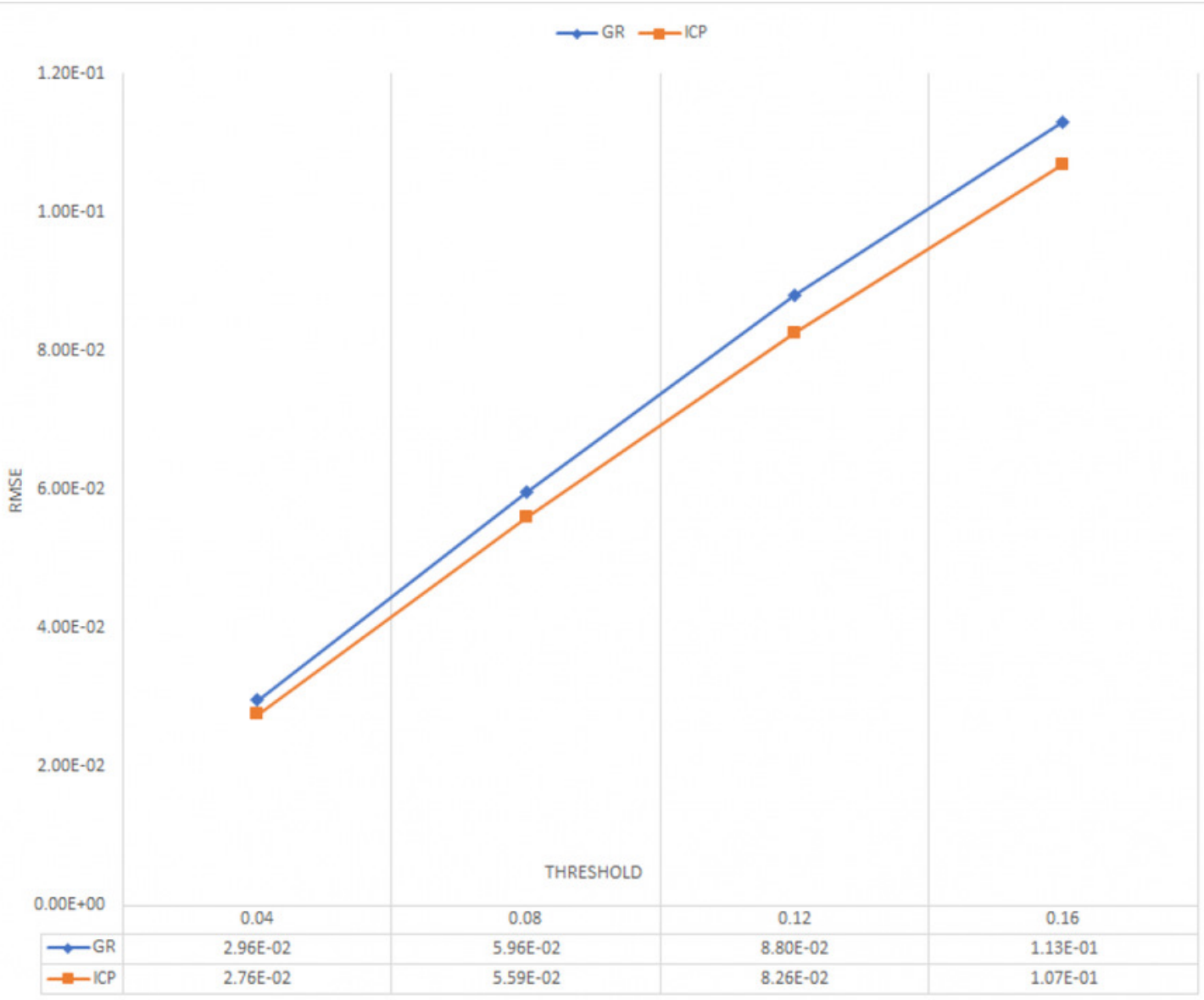


Figure 7

Average Fitness for 20 frames

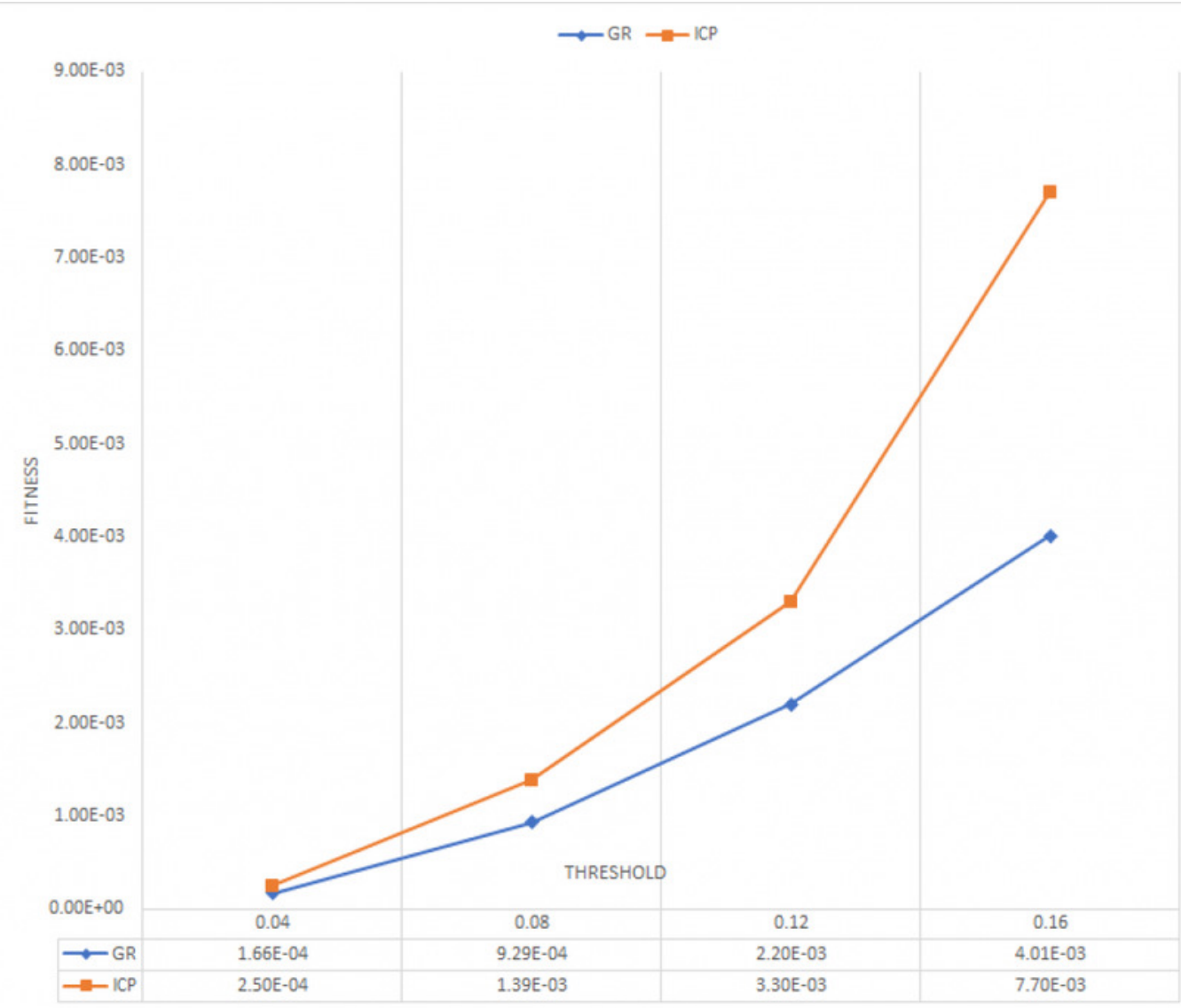


Figure 8

\section{ICP-RMSE for 20 frames}

$1.20 \mathrm{E}-01$

1.00E-01

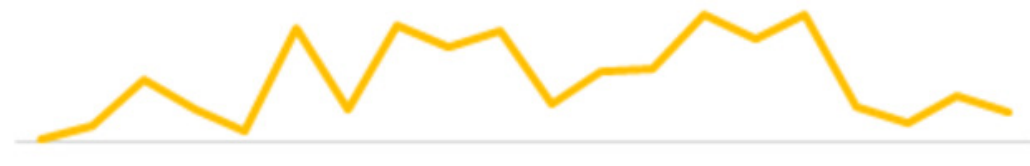

8.00E-02

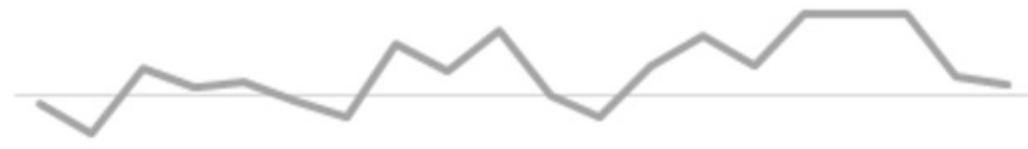

$\sum_{\substack{n \\:}}^{W} 6.00 E-02$

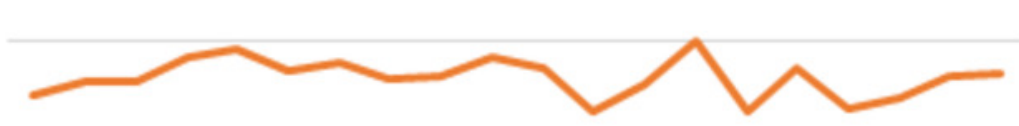

- RMSE $(r=0.04$ STDV $=0.0016)$

RMSE( $r=0.08$ STDV $=0.002$ )

- RMSE( $r=0.12$ STDV=00.39) $-\operatorname{RMSE}(r=0.16$ STDV=00.46)

4.00E-02

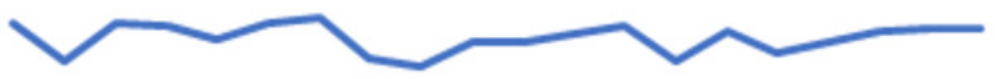

2.00E-02

$0.00 E+00$

$\begin{array}{llllllllllllllllllll}1 & 2 & 3 & 4 & 5 & 6 & 7 & 8 & 9 & 10 & 11 & 12 & 13 & 14 & 15 & 16 & 17 & 18 & 19 & 20 \\ \text { frames number }\end{array}$ 
Figure 9

3D point cloud mapped to 2D KITTI image (Geiger, 2013)

(a) selected input frame (b) Ground truth (c) predicted points

KITTI dataset is under the Creative Commons Attribution-NonCommercial-ShareAlike 3.0 License. according to this link: http://www.cvlibs.net/datasets/kitti/
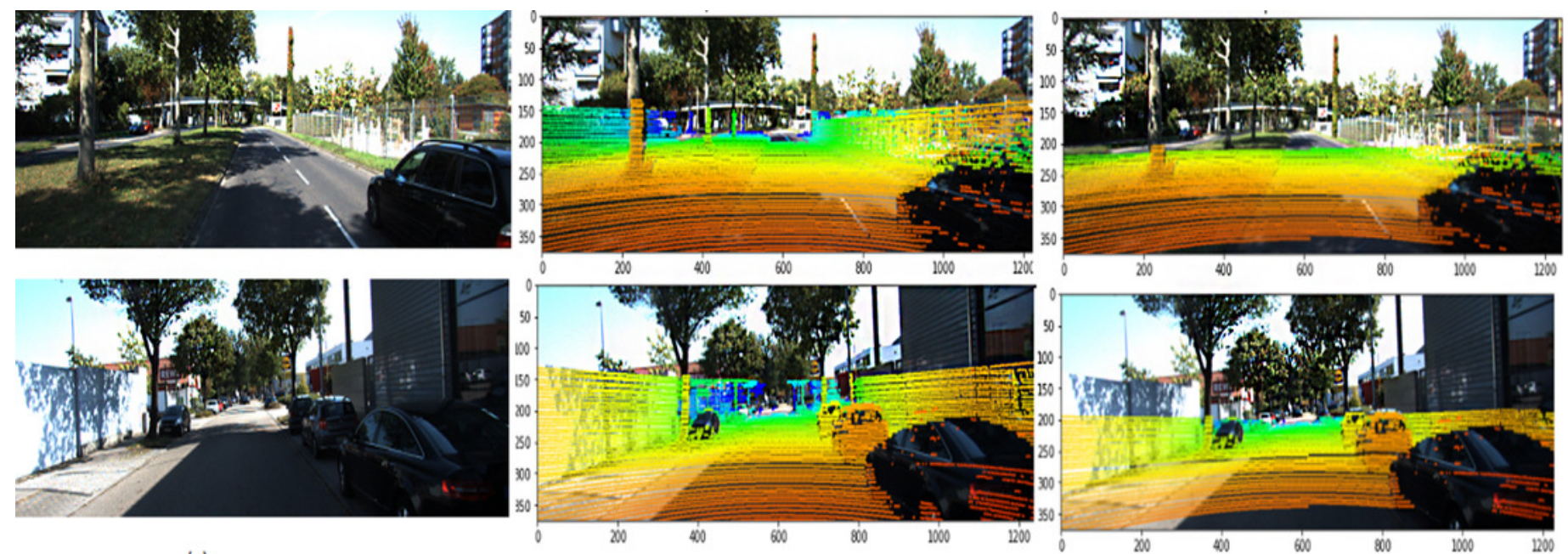

(a)

(b)

(c) 
Figure 10

MRE for 3D reconstruction using different techniques on KITTI dataset

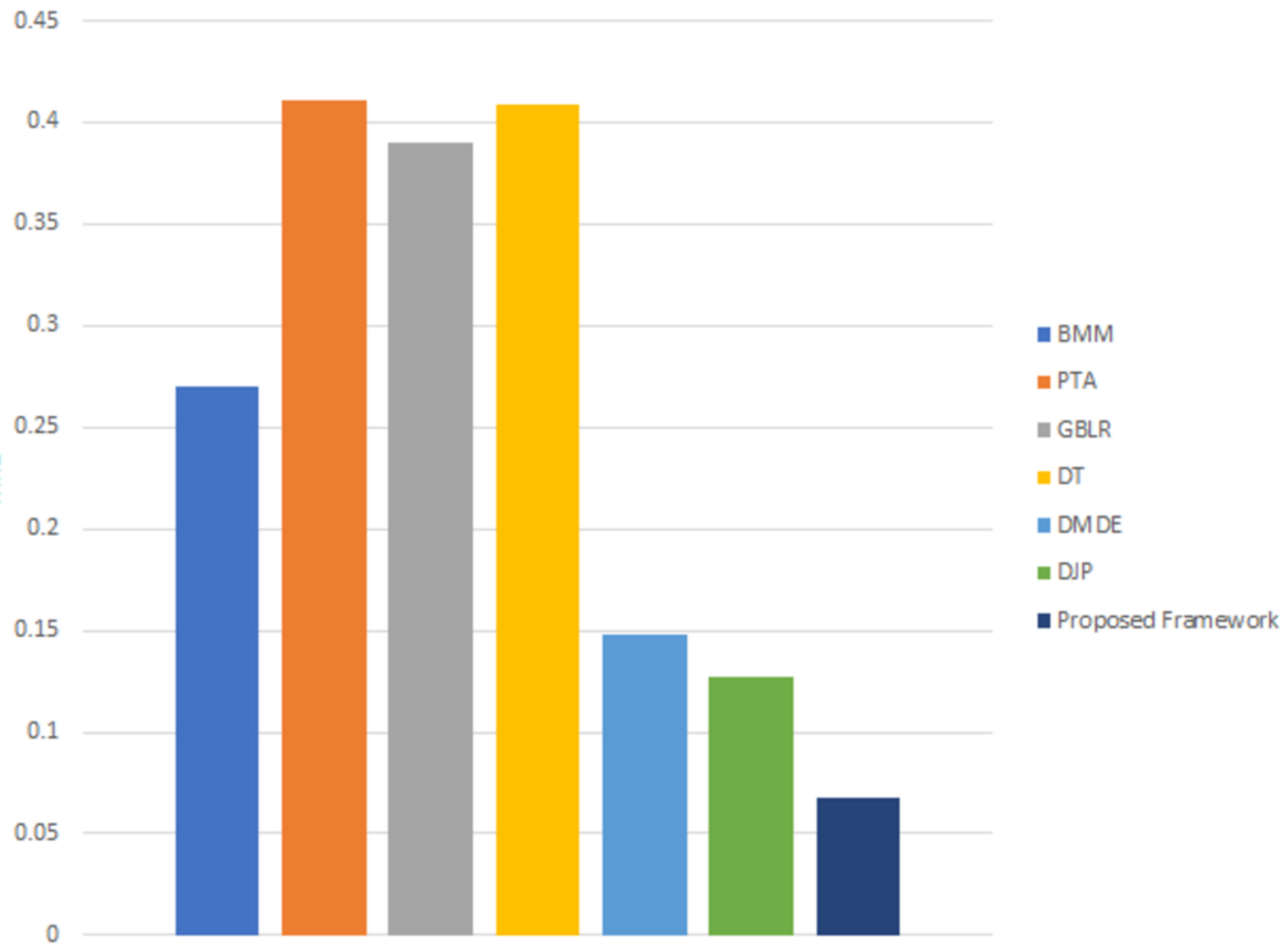




\section{Table $\mathbf{1}$ (on next page)}

The main characteristics of the most relevant State-of-the-art 
1 Table 1: The main characteristics of the most relevant State-of-the-art

\begin{tabular}{|c|c|c|c|c|c|}
\hline Published & $\begin{array}{c}\text { Single } \\
\text { /Multiple } \\
\text { frame }\end{array}$ & $\begin{array}{c}\text { Single } \\
\text { /Multiple } \\
\text { object }\end{array}$ & $\begin{array}{c}\text { Static / } \\
\text { Dynamic } \\
\text { object }\end{array}$ & Input type & Methods \\
\hline (Kulikajevas, 2019) & Single frame & Single object & Static object & $\begin{array}{c}\text { RGB-D } \\
\text { sensor }\end{array}$ & $\begin{array}{l}\text { Hyper neural } \\
\text { network }\end{array}$ \\
\hline (Kulikajevas, 2019) & Single frame & Single object & Static object & 3D Models & $\begin{array}{l}\text { GANs neural } \\
\text { network }\end{array}$ \\
\hline (Widya, 2019) & $\begin{array}{l}\text { Multiple (2 } \\
\text { image } \\
\text { sequences) }\end{array}$ & Single object & Static object & $\begin{array}{l}\text { Monocular } \\
\text { endoscope }\end{array}$ & $\begin{array}{c}\text { Structure from } \\
\text { motion (SfM) }\end{array}$ \\
\hline (Wang J. H., 2018) & Single frame & Single object & Static object & $\begin{array}{c}\text { RGB-D } \\
\text { sensor }\end{array}$ & $\begin{array}{l}\text { Monocular } \\
\text { SLAM }\end{array}$ \\
\hline (Yang X. L., 2020) & Single frame & $\begin{array}{l}\text { Multiple } \\
\text { (full scene) }\end{array}$ & $\begin{array}{l}\text { Static scene } \\
\text { (remove } \\
\text { dynamic } \\
\text { objects) }\end{array}$ & $\begin{array}{l}\text { Monocular } \\
\text { RGB }\end{array}$ & $\begin{array}{c}\text { Online } \\
\text { incremental } \\
\text { mesh } \\
\text { generation }\end{array}$ \\
\hline (Shimada, 2020) & Single frame & Single object & $\begin{array}{c}\text { Dynamic } \\
\text { object }\end{array}$ & $\begin{array}{l}\text { Monocular } \\
\text { RGB }\end{array}$ & $\begin{array}{l}\text { Markless 3D } \\
\text { human motion } \\
\text { capture }\end{array}$ \\
\hline (Peng, 2020) & Single frame & Single object & $\begin{array}{l}\text { Dynamic } \\
\text { object }\end{array}$ & $\begin{array}{l}\text { Monocular } \\
\text { RGB }\end{array}$ & GCN network \\
\hline (Ku, 2019.) & Single frame & $\begin{array}{c}\text { Corp single } \\
\text { object }\end{array}$ & $\begin{array}{c}\text { Dynamic } \\
\text { object }\end{array}$ & $\begin{array}{l}\text { Monocular } \\
\text { RGB }\end{array}$ & $\begin{array}{l}\text { geometric } \\
\text { priors, shape } \\
\text { reconstruction, } \\
\text { and depth } \\
\text { prediction }\end{array}$ \\
\hline$(\mathrm{Lu}, 2020)$ & $\begin{array}{l}\text { Multiple (two } \\
\text { consecutive } \\
\text { point-cloud) }\end{array}$ & $\begin{array}{l}\text { Multiple } \\
\text { (full scene) }\end{array}$ & $\begin{array}{l}\text { Dynamic } \\
\text { objects }\end{array}$ & $\begin{array}{l}\text { Outdoor } \\
\text { LiDAR } \\
\text { datasets }\end{array}$ & $\begin{array}{l}\text { LSTM and } \\
\text { GRU networks }\end{array}$ \\
\hline (Weng, 2020. ) & Single frame & $\begin{array}{l}\text { Multiple } \\
\text { (full scene) }\end{array}$ & $\begin{array}{c}\text { Dynamic } \\
\text { objects }\end{array}$ & $\begin{array}{l}\text { Outdoor } \\
\text { LiDAR } \\
\text { datasets }\end{array}$ & $\begin{array}{l}\text { Predict next } \\
\text { scene using } \\
\text { LSTM }\end{array}$ \\
\hline (Akhter, 2010) & Single frame & $\begin{array}{l}\text { Multiple } \\
\text { objects }\end{array}$ & $\begin{array}{l}\text { Dynamic } \\
\text { objects }\end{array}$ & $\begin{array}{l}\text { Monocular } \\
\text { RGB }\end{array}$ & $\begin{array}{l}\text { Structure from } \\
\text { motion }\end{array}$ \\
\hline
\end{tabular}




\begin{tabular}{|c|c|c|c|c|c|}
\hline (Fragkiadaki, 2014) & $\begin{array}{l}\text { Multiple } \\
\text { frames }\end{array}$ & Single object & $\begin{array}{c}\text { Dynamic } \\
\text { object }\end{array}$ & $\begin{array}{l}\text { Monocular } \\
\text { RGB }\end{array}$ & $\begin{array}{c}\text { non-rigid } \\
\text { structure-from- } \\
\text { motion } \\
(\mathrm{NRSfM})\end{array}$ \\
\hline (Ranftl, 2016) & $\begin{array}{c}\text { Multiple } \\
\text { frames (two } \\
\text { consecutive) }\end{array}$ & $\begin{array}{l}\text { Multiple } \\
\text { (full scene) }\end{array}$ & $\begin{array}{c}\text { Dynamic } \\
\text { object }\end{array}$ & $\begin{array}{c}\text { Monocular } \\
\text { RGB }\end{array}$ & $\begin{array}{l}\text { segments the } \\
\text { optical flow } \\
\text { field into a set } \\
\text { of motion } \\
\text { models }\end{array}$ \\
\hline Kumar S. D., 2019) & $\begin{array}{l}\text { Multiple (2 } \\
\text { frames) }\end{array}$ & $\begin{array}{l}\text { Multiple } \\
\text { (full scene) }\end{array}$ & $\begin{array}{c}\text { Dynamic } \\
\text { objects }\end{array}$ & $\begin{array}{c}\text { Monocular } \\
\text { RGB }\end{array}$ & $\begin{array}{l}\text { Super pixel } \\
\text { over } \\
\text { segmentation }\end{array}$ \\
\hline Proposed framework & $\begin{array}{c}\text { Multiple } \\
\text { (whole video } \\
\text { frames } \\
\text { sequence) }\end{array}$ & $\begin{array}{l}\text { Multiple } \\
\text { (full scene) }\end{array}$ & $\begin{array}{l}\text { Dynamic } \\
\text { objects }\end{array}$ & $\begin{array}{l}\text { Monocular } \\
\text { RGB }\end{array}$ & $\begin{array}{l}\text { Unsupervised } \\
\text { learning and } \\
\text { point cloud } \\
\text { fusion }\end{array}$ \\
\hline
\end{tabular}




\section{Table 2 (on next page)}

The improvement percentages in MRE error between proposed framework and the state-of-the-art 
8 Table 2:

9 The improvement percentages in MRE error between proposed framework and the state-of10 the-art

11

\begin{tabular}{|l|l|}
\hline Approach & $\begin{array}{l}\text { Improvement } \\
\text { Percentage } \%\end{array}$ \\
\hline BMM & 75.02775 \\
\hline PTA & 83.58463 \\
\hline GBLR & 82.70561 \\
\hline DT & 83.49633 \\
\hline DMDE & 54.39189 \\
\hline DJP & 46.76656 \\
\hline
\end{tabular}




\section{Table 3 (on next page)}

Comparison using Chamfer Distance between the proposed framework and state-of-theart 
1

2

3

4

5

6

7

8

9 Table 3:

10 Comparison using Chamfer Distance between the proposed framework and state-of-the-art

\begin{tabular}{|l|l|}
\hline \multicolumn{2}{|c|}{ KITTI } \\
\hline Model & Chamfer Distance \\
\hline MoNet (LSTM) & 0.573 \\
MoNet (GRU) & 0.554 \\
Proposed framework & $\mathbf{0 . 4 9 1}$ \\
\hline
\end{tabular}

